\title{
Child mental health problems in paediatric practice: the hidden psychopathology
}

\author{
Hemamali Perera ${ }^{1}$ \\ Sri Lanka Journal of Child Health, 2003; 32: 64-5
}

(Key words: mental health problems, child)

Behavioural and emotional problems have been identified in $18-20 \%$ of children attending paediatric primary care ${ }^{1}$. Similar problems, but in a higher prevalence of $28 \%$, are reported from specialist paediatric ambulatory care services ${ }^{2}$. As many as two thirds of randomly selected hospitalised children have been found to have psychological difficulties ${ }^{3}$.

At one end of the spectrum are children with longterm physical disorders and associated psychological disturbances. This includes children with almost any kind of chronic illness, neurological disease, disfiguring and life threatening illness, recurrent hospitalisation and those subjected to invasive procedures. All these children run a high risk of developing mental health problems ${ }^{4}$. When the psychological impact of individual illnesses is considered, a rich source of data is available on children suffering from epilepsy, asthma, diabetes and cancer. Apart from the general psychosocial difficulties associated with illness and disability, poor emotional adjustment to chronic or life threatening illness is known to jeopardise disease control and the compliance with treatment regimes ${ }^{4,5}$. Presence of psychological symptoms may also complicate the diagnostic process, promote longer hospitalisation and increase health care $\operatorname{cost}^{6}$. Some international classifications of diseases even provide guidelines for diagnosing and coding of such secondary psychological effects of physical disorders ${ }^{7}$.

At the opposite end of the spectrum are children who present with somatic symptoms, which at first glance may suggest a physical disorder, but where there is no evidence of this. These medically unexplained physical presentations are common in paediatric practice and prevalence as high as $25 \%$ is known ${ }^{8}$. Here, the psychological distress is masked by a physical complaint or expressed through a bodily symptom. This is not surprising as emotions have

\footnotetext{
${ }^{1}$ Consultant Psychiatrist, Department of Psychological Medicine, Faculty of Medicine, University of Colombo.
}

physical correlates. Recurrent abdominal pain, headache and failure to thrive of non-organic nature are examples of this where an underlying depression and anxiety related to external stresses can often be elicited. There is excessive use of health care services in these cases, with an additional burden on resources from expensive and exhaustive investigations, but with negative results ${ }^{9}$.

Despite all the evidence available, a major difficulty in satisfactory management of such symptoms and psychopathology is that they remain largely unrecognised $^{10,11}$. On the one hand, overcrowded clinics and inadequate provision of consultation time is often blamed for the failure to recognise such symptoms. At the same time, under-emphasis of the importance of mental health and behaviour problems in some paediatric training programmes will leave a deficiency in knowledge about the existence of such psycho-pathology and skills in eliciting them ${ }^{12}$. There are other obstacles including an ambivalent attitude and stereotyped attributions to psychological matters, stigma, prejudice and anxiety about dealing with emotional problems, which would prevent the doctor from pursuing a productive psychosocial inquiry. On the other hand, the parents and the child may also contribute to poor recognition of these problems. It is known that parents are reluctant to voice psychosocial concerns to the doctor unless inquiry is actively pursued $^{13,14}$. Children too have been found to report fewer symptoms of depression, which may reflect downplaying, minimising and denial of distress or difficulty in finding words to describe emotions ${ }^{15}$.

Successful management of mental health problems in paediatric practice requires an ability to identify psychopathology and understand risk factors that predispose children to such disturbances. Sleep and appetite changes, anxiety and phobic symptoms, irritability, aggression and emotional and behavioural regression are some common symptoms to look for. Risk factors that make children with a physical disorder more vulnerable too are well known ${ }^{16}$. Non- 
coping and distressed parents who have a poor understanding of the child's illness, inadequate psychological preparation for invasive procedures, lack of support and appropriate response from healthcare staff and multiple medical consultations with conflicting opinions are such risk factors. It is clear that some of these risk factors are preventable and that support for acceptance and positive adaptation to illness will improve outcome oftreatment ${ }^{17}$. Most current guidelines in health care practices emphasize a holistic approach to medical management, where all aspects of health needs and quality of life are addressed. The benefit of such total care to children and families is well recognised. Thus, two recommendations that could be made towards achieving this benefit are to include basic psychological and behavioural aspects in paediatric training to improve recognition and intervention with mental health problems and a closer collaboration between child mental health and paediatric services for consultation and referral in more complex and severe cases.

\section{References}

1. Costello E J, Costello A J, Edelbrock C. Burns B J et al. Psychiatric disorders in paediatric primary care. Archives of General Psychiatry 1988; 45:1107-16.

2. Garralda M E, Bailey D. Psychiatric disorders in general paediatric referrals. Archives of Diseases of Childhood 1989; 64:1727-33.

3. Shugart M A. Child psychiatry consultation to inpatients: a literature review. General Hospital Psychiatry 1991; 13: 325-36.

4. Cadman D, Boyle M, Szatmari P, Offord D R. Chronic illness, disability, mental and social well being: findings of the Ontario Child Health Study. Pediatrics 1987; 79:805-18.

5. Northam E A. Psychosocial impact of chronic illness in children. Journal of Paediatrics and Child Health 1997; 33: 369-72.

6. Steiner H, Fritz G K, Mrazek D. Paediatric and psychiatric comorbidity. Part I: the future of consultation-liaison psychiatry. Psychosomatics 1993; 34:107-11.

7. Diagnostic and Statistical Manual of Mental Disorders 4th ed. American Psychiatric Association 1994; Washington DC.
8. Brill S R, Patel D R, MacDonald E. Psychosomatic disorders in paediatrics. Indian Journal of Pediatrics 2001; 68: 597-603.

9. Kelleher K J, Starfield B. Health care use by children receiving mental health services. Pediatrics 1990; 85:114-8.

10. Horowitz Identification of psychosocial problems in paediatric primary care. Pediatrics 1992; 89:4805 .

11. Dulcan M K, Costello A J, Edelbrock C, Brent S. Janiszewski S. The pediatrician as gatekeeper to mental health care in children: do parents' concern open the gate? Journal of the American Academy of Child and Adolescent Psychiatry 1990; 29:453-58.

12. Graham P. Mental health should be centre stage in child welfare. Archives of Diseases of Childhood 2000; 83: 4-7.

13. Cassidy L J, Jellinek M S. Approaches to recognition and management of childhood psychiatric disorders in paediatric primary care. Pediatric Clinics of North America 1998; 45: 1037 52.

14. Barry C A, Bradley C P, Britten N, Stevenson F A, Barber N. Parents' unvoiced agendas in general practice consultations: qualitative study. British Medical Journal 2000; 320: 1246-50.

15. Kaplan S L, Busner J, Weinhold C, Lenon P. Depressive symptoms in children and adolescents with cancer: a longitudinal study. Journal of the American Academy of Child and Adolescent Psychiatry 1987; 26:782-87.

16. Lewis M, Leebens $\mathrm{P} \mathrm{K}$. The consultation process in child and adolescent consultation - liaison in paediatrics. In: Lewis M. Child and Adolescent Psychiatry, A Comprehensive Textbook 2nd ed. 1996; Williams and Wilkins, Baltimore. 935-9.

17. Wamboldt M Z, Wamboldt F S. Role of family in onset and outcome of childhood disorders: selected research findings. Journal of the American Academy of Child and Adolescent Psychiatry 2000; 39:1212-19. 
